\title{
Long-term effects of Graves' ophthalmopathy on health-related quality of life
}

\author{
Caroline Terwee ${ }^{1,4}$, Iris Wakelkamp ${ }^{2}$, Stevie Tan ${ }^{3}$, Friedo Dekker ${ }^{5}$, Mark F Prummel ${ }^{2}$ and Wilmar Wiersinga ${ }^{2}$ \\ ${ }^{1}$ Department of Clinical Epidemiology and Biostatistics, ${ }^{2}$ Department of Clinical Endocrinology and Metabolism, and ${ }^{3}$ Orbital Centre, Academic Medical \\ Centre, University of Amsterdam, ${ }^{4}$ Institute for Research in Extramural Medicine, VU Medical Centre, Amsterdam and ${ }^{5}$ Department of Clinical \\ Epidemiology, Leiden University Medical Centre, The Netherlands
}

(Correspondence should be addressed to C Terwee; Email: cb.terwee.emgo@med.vu.nl)

\begin{abstract}
Objective: Little is known about the long-term effects of Graves' ophthalmopathy (GO) on healthrelated quality of life (HRQL) after the eye treatment is considered to be finished. The aim of this study was to quantify these effects using validated HRQL questionnaires.

Design: A cross-sectional follow-up study was carried out in GO patients who had started radiotherapy and/or prednisone treatment between 1982 and 1992.

Methods: Between 1998 and 2000 these patients received an HRQL questionnaire containing the SF36, EuroQol and GO-QOL questionnaires. All patients were invited for a follow-up ophthalmological examination. HRQL scores of the respondents were compared with those of two reference populations of 'healthy' persons and to scores of several other GO populations.

Results: One hundred and sixty-eight patients were included; 163 completed the HRQL questionnaire and 154 visited the outpatient clinic. The median follow-up was 11.7 years. A considerable number of symptoms related to GO were found. More than half of the patients had diplopia and $28 \%$ had a low visual acuity. Sixty per cent had a proptosis of at least $20 \mathrm{~mm}$. The HRQL scores were considerably better than those of newly diagnosed GO patients (untreated) and GO patients who completed the questionnaire during treatment, but worse than those of 'healthy' persons. Although we did not perform a longitudinal study, we included a selected group of patients who had been treated with radiotherapy and/or prednisone in the Academic Medical Centre of the University of Amsterdam, and though we could not assess HRQL at comparable times after receiving treatment, this is the first study that presents any data on the long-term effects of GO on HRQL.

Conclusion: GO has a marked negative effect on HRQL, even many years after treatment. These findings suggest that GO should be considered a chronic disease. Aftercare is needed for these patients after their immunosuppressive and surgical treatments.
\end{abstract}

European Journal of Endocrinology 146 751-757

\section{Introduction}

Graves' ophthalmopathy (GO) is characterised by symptoms such as pain, grittiness, and excessive tearing; visual limitations as a result of reduced vision and diplopia, and cosmetic changes due to proptosis, eyelid retraction and swelling (1). Although the impact of these changes on the patients' lives have always been acknowledged in clinical practice, only recently have the effects of $\mathrm{GO}$ on health-related quality of life (HRQL) been quantified using validated questionnaires. GO patients reported marked limitations in multiple aspects of physical and mental functioning, compared with patients without any chronic condition (2). Using a disease-specific questionnaire, $35 \%$ of the patients reported limitations in driving and performing leisure activities, $71 \%$ felt their eye disease had influ- enced their self-confidence and $40 \%$ felt socially isolated because of their GO (3).

Little is known about the long-term effects of GO on HRQL after the final treatment. Bartley et al. (4) reported a median duration of treatment for mild GO patients of about 4 years. After a follow-up of about 6 years since the final examination, $52 \%$ of the respondents felt that their eyes were still abnormal and 38\% were unsatisfied with the appearance of their eyes (4). These data suggest that GO might be a lifelong chronic condition. However, follow-up studies using validated HRQL instruments are lacking.

Recently, we carried out a cross-sectional follow-up study to examine the risks of developing retinopathy in patients who had started radiotherapy and/or prednisone treatment for GO about 12 years ago. This study gave us the opportunity to evaluate the long-term 
effects of GO on HRQL after the eye treatment was considered to be finished, using validated HRQL questionnaires.

\section{Subjects and methods}

\section{Patients and procedures}

A cross-sectional follow-up study was carried out in 1998-2000 in patients who had started radiotherapy and/or prednisone treatment for GO between 1982 and 1992 at the Academic Medical Centre (AMC) in Amsterdam. Patients were identified by reviewing all consultations recorded in the diaries of the Orbital Centre. Inclusion criteria were a diagnosis of GO and treatment with orbital radiotherapy and/or prednisone between 1982 and 1992. The study was approved by the medical ethics committee of the AMC. Addresses of the eligible patients were retrieved from their medical records. Patients were asked for informed consent by a mailed letter. Non-responders were called by a nurse or secretary. Patients who still refused to participate were called a second time by an ophthalmologist who explained the importance of the study one more time. If the address of a patient was incorrect or unknown, the current address was retrieved at first from the general practitioner and eventually from the district council.

Respondents who returned the informed consent received a questionnaire by mail and were invited to the outpatient clinic of the Orbital Centre of the AMC for an ophthalmological examination. The questionnaire contained the SF-36 Health Survey (5) and the EuroQol (6) as well as the GO-QOL (3). The SF-36 and EuroQol are both general HRQL questionnaires, assessing limitations in physical and psychosocial functioning as a consequence of any health condition. The EuroQol also includes a visual analogue scale (VAS), which is a line (sometimes called a thermometer) on which subjects can indicate how good or how bad their current health (in general) is, according to their opinion. These questionnaires were chosen because they have also been used in other GO patient groups, with whom the data can be compared (see below).

The GO-QOL is a disease-specific HRQL questionnaire, specifically developed for patients with GO. The GO-QOL consists of 16 questions, eight questions referring to limitations in visual functioning as a consequence of decreased visual acuity and/or diplopia (e.g. problems with driving, reading, hobbies), and eight questions referring to limitations in psychosocial functioning as a consequence of a changed appearance (e.g. feeling socially isolated, influence on self-confidence). In addition, questions were included about the kind of treatments for GO that the patients had received since diagnosis and about existing comorbidity. The presence of comorbidity was defined as controlled, but mildly symptomatic disease (IDS2 or higher) according to Greenfield et al. (7).

At the outpatient clinic, the following disease characteristics were assessed: best corrected visual acuity, soft tissue involvement, lid aperture, proptosis (all presented in the worst eye), diplopia according to the Gorman score $(0=$ no diplopia; $1=$ intermittent diplopia; $2=$ inconstant diplopia, only in certain directions of gaze; $3=$ constant diplopia in primary and/or reading position), and current GO activity according to the seven-point Clinical Activity Score (CAS) (8). The CAS is based on seven signs of inflammation of the orbit: pain, redness and swelling, each scored as present (score 1) or absent (score 0) (8).

\section{Statistical analyses}

The duration of follow-up was calculated as the time between the first visit to the Orbital Centre at the AMC for GO and the follow-up visit for this study. The 16 GO-QOL questions were scored as 'severely limited', 'a little limited', or 'not limited at all' and summarised into one total score for visual functioning and one total score for appearance (3). The general HRQL questionnaires were scored as proposed by their authors $(5,6$, 9). All HRQL scales were transformed to a score ranging from 0-100 (higher scores indicating better health).

\section{Comparison of HRQL scores with other populations}

To examine if our cohort of GO patients (in the tables referred to as 'GO patients after treatment') were still limited in their general health compared with 'healthy' people, we compared their scores on the SF-36 with SF36 scores of a reference population of 1742 Dutch persons, drawn at random from the national telephone registry (10). Mean \pm S.D. age of this population was $48 \pm 18$ years, $54 \%$ were female, $13 \%$ had primary education, $62 \%$ had secondary education and $25 \%$ went to college/university. In addition, scores were compared with a convenience sample of 206 GO patients who completed the SF-36 before the start of radiotherapy or orbital surgery in 1997-1998 (11) (referred to as 'GO patients during treatment').

For this purpose, Z-scores were computed for both GO patient groups by dividing the difference between the mean SF-36 score of the patient group and the mean SF-36 score of the reference population by the S.D. of the mean SF-36 score of the reference population. Because of the large difference in percentage of women between the GO groups and the reference population, Z-scores were adjusted for gender, by using gender-specific SF-36 scores of the reference population.

In addition, a physical component score (PCS) and a mental component score (MCS) were calculated for all 
Table 1 General characteristics of the patient group and comparison groups (values are percentages, except those for years and months).

\begin{tabular}{|c|c|c|c|c|}
\hline & $\begin{array}{l}\text { GO patients } \\
\text { after treatment }\end{array}$ & $\begin{array}{l}\text { GO patients } \\
\text { before treatment }\end{array}$ & $\begin{array}{l}\text { GO patients } \\
\text { during treatment }\end{array}$ & $\begin{array}{l}\text { GO patients } \\
\text { mixed sample }\end{array}$ \\
\hline General characteristics at the time of questionnaire completion & $n=168$ & $n=70(3)$ & $n=206(11)$ & $n=93(14)$ \\
\hline Age (years), mean \pm S.D. & $58 \pm 13$ & $53 \pm 13$ & $50 \pm 12$ & $49 \pm 14$ \\
\hline Sex, F/M & $78 / 22$ & $71 / 29$ & $83 / 17$ & $75 / 25$ \\
\hline Education, primary/secondary education/college or university & $50 / 38 / 12$ & $42 / 44 / 14$ & $34 / 50 / 16$ & 29/52/19 \\
\hline Comorbidity ${ }^{1}$, yes/no & $26 / 74$ & n.a. & n.a. & n.a. \\
\hline Duration of follow-up ${ }^{3}$ (years), median (range) & $11.7(7.4-23.4)$ & - & - & - \\
\hline Duration of $\mathrm{GO}^{3}$ (months), median (range) & - & $12(1-206)$ & $39(4-571)$ & $45(4-364)$ \\
\hline Immunosuppression, radiotherapy/prednisone/both & $13 / 32 / 55$ & $0 / 0 / 0$ & $5 / 29 / 16$ & 30/50/n.a. \\
\hline $\begin{array}{l}\text { Orbital surgery, decompression/eye muscle surgery/eyelid } \\
\text { surgery }\end{array}$ & $41 / 53 / 52$ & $0 / 0 / 0$ & $27 / 24 / 17$ & $57 / 39 / 45$ \\
\hline \multicolumn{4}{|l|}{ Current GO severity } & $n=93$ \\
\hline Soft tissue involvement, no/mild/moderate/severe & $87 / 12 / 1 / 0$ & $9 / 60 / 27 / 4$ & $16 / 40 / 34 / 10$ & $15 / 59 / 25 / 1$ \\
\hline Proptosis $(\mathrm{mm}),<16 / 16-19 / 20-23 />23$ & $8 / 32 / 46 / 14$ & $0 / 0 / 74 / 26$ & $16 / 46 / 27 / 11$ & $0 / 0 / 78 / 22$ \\
\hline Lid aperture $(\mathrm{mm}),<9 / 9-11 />11$ & $12 / 75 / 13$ & n.a. & $3 / 27 / 70$ & $5 / 62 / 33$ \\
\hline Diplopia, no/intermittent/inconstant/constant & $49 / 16 / 25 / 10$ & $16 / 37 / 43 / 4$ & $37 / 12 / 21 / 30$ & $53 / 25 / 22 / 0$ \\
\hline $\begin{array}{l}\text { Visual acuity (Snellen line) }, \geq 0.80 / \geq 0.63-0.80 / \geq 0.40- \\
0.63 /<0.40\end{array}$ & $72 / 17 / 6 / 5^{2}$ & $96 / 0 / 0 / 4$ & $78 / 10 / 8 / 4$ & $90 / 0 / 4 / 6$ \\
\hline \multicolumn{5}{|l|}{ Current GO activity } \\
\hline CAS, $0 / 1-4 / 5-7$ & $74 / 26 / 0$ & $1 / 90 / 9$ & $12 / 75 / 13$ & $38 / 52 / 10$ \\
\hline
\end{tabular}

GO patients in both groups, based on the Z-scores, according to Ware et al. (12). The PCS and MCS scores represent the deviation from the reference population expressed in S.D.s of the reference population. The reference population has a mean PCS and MCS score of 50 , with an S.D. of 10 . We calculated the percentage of respondents who scored 2 S.D. below the reference population, i.e. a score of $<30$ points on the PCS and MCS. These scores reflect the lowest 2.5 percentile of the reference population.

The EuroQol scores of our patients were compared with those of the same GO patient population 'during treatment' and to a specific Dutch reference population for the EuroQol, described by Essink-Bot et al. (13). These comparisons could not be adjusted for genderdifferences because no gender-specific data were available of the reference population (13).

Scores of the GO-QOL were compared with scores of three other GO patient populations for which GO-QOL scores were available. These groups were: (i) a group of 70 newly diagnosed GO patients, visiting the Orbital Centre of the AMC for the first time (3) (in the tables referred to as 'GO patients before treatment') - these patients were untreated for their GO; (ii) the beforementioned convenience sample of 206 GO patients who completed the GO-QOL during treatment ('GO patients during treatment'); and (iii) a group of all consecutive patients $(n=93)$ who visited the Orbital Centre during a 3 month period (14) ('GO mixed sample'). This group is a mixture of patients who completed the GO-QOL before, during, or after treatment. Characteristics of all GO patient groups are presented in Table 1.

\section{Results}

\section{Response and patient characteristics}

Two hundred and forty-seven patients started radiotherapy or prednisone therapy between 1982 and 1992 at the AMC. Of this cohort, 34 patients were deceased at the time of follow-up (none because of GO-related causes), leaving 213 eligible participants for this study. Despite two reminders by telephone, 45 patients refused to participate, leaving 168 patients, who were included in the study. The response rate was $79 \%$ (168 out of 213). Of the 168 included patients, 14 patients only completed the questionnaire, but refused to visit the outpatient clinic, and five patients refused or were unable to complete the questionnaire, but did visit the outpatient clinic. In total, 163 HRQL questionnaires were completed and 154 patients visited the Orbital Centre.

Patient characteristics are presented in Table 1. The median follow-up after the first visit to the AMC was 11.7 years (range 7.4-23.4). Thirteen per cent of the patients were treated with radiotherapy, 32\% with prednisone and 55\% with both immunosuppressive treatment modalities. The median number of orbital operations that patients had undergone was two 


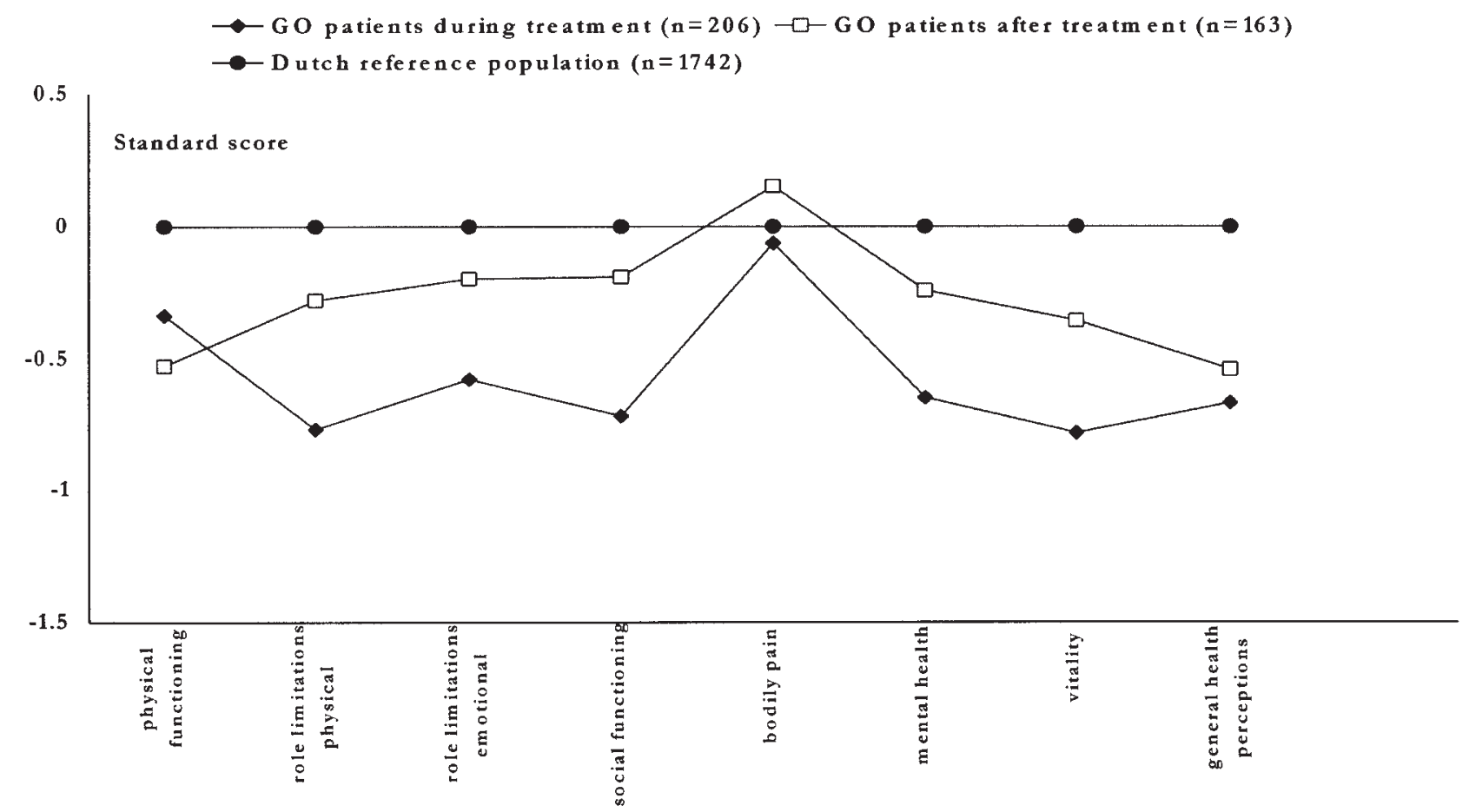

Figure 1 SF-36 Z-scores of GO patients from this study $(n=163)$ compared with previously treated GO patients $(n=206)$ and a Dutch reference population $(n=1742)$.

(range 0-13). Sixty per cent of the patients had a proptosis of at least $20 \mathrm{~mm}$, and $14 \%$ had a proptosis of at least $23 \mathrm{~mm}$. Fifty-one per cent of the patients had diplopia and $28 \%$ of the patients had a visual acuity below 0.8. One patient was almost blind due to the GO. Twenty-six per cent of the patients had a CAS $>0$.

\section{HRQL}

In Fig. 1 the results of the SF-36 questionnaire are presented. Patients in our cohort reported on average significantly fewer limitations $(P<0.05$ to $P<0.001)$ compared with a comparison population of GO patients who completed the questionnaire during treatment, except for physical functioning and general health perceptions. Patients reported more limitations compared with the reference population of 'healthy' persons on all general HRQL measures $(P<0.001)$, except for bodily pain. The largest differences with the reference population were found for general health perceptions and physical functioning (Z-scores -0.54 and -0.53 respectively) (Fig. 1). The mean PCS was $46.5 \pm 11.3$

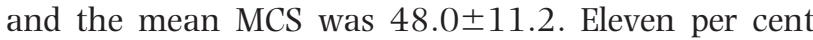
of the patients in our cohort scored a PCS of $<30$ points and $9 \%$ scored an MCS of $<30$ points. In the cohort of GO patients during treatment the mean PCS was $45.9 \pm 10.2$ and the mean MCS was 41.0 \pm 13.4 . Seven per cent of the patients scored a PCS of $<30$ points and $26 \%$ of this cohort scored an MCS $<30$ points.

On the EuroQol questionnaire, our patients scored similarly to the GO patients who completed the questionnaire during treatment with respect to mobility, self-care and pain, and significantly higher (better) with respect to usual activities and anxiety $(P<$ $0.001)$, and overall health perception (VAS) $(P<$ 0.001). The mean scores of our patients were lower than the mean scores of the reference populations on all dimensions. A mean difference of 14 points was found between our GO patients and the reference population on the VAS $(P<0.001$, Table 2$)$.

In Table 3, GO-QOL scores are presented. On average, patients in our cohort reported less limitations (i.e. significantly higher scores, $P<0.001$ ) compared with the three GO comparison groups. Compared with newly diagnosed GO patients (before treatment) our patients scored 23.5 points higher on visual functioning $(P<$ $0.001)$ and 17.1 points on appearance $(P<0.001)$.

Because the GO-QOL specifically measures limitations in functioning as a result of GO, 'healthy' persons should score 100 points (no limitations). In this cohort, $30 \%$ of the patients scored 100 points for visual functioning and 19\% scored 100 points on appearance. Fifty-seven and $61 \%$ of the patients scored below 90 points (in a previous study a mean difference of 10 points was considered a clinically important difference (11)) and 12 and $13 \%$ of the 
Table 2 General HRQL scores: the EuroQol questionnaire.

\begin{tabular}{|c|c|c|c|}
\hline & $\begin{array}{l}\text { GO patients during } \\
\text { treatment }(11) \\
\quad(n=204)^{1}\end{array}$ & $\begin{array}{l}\text { GO patients } \\
\text { after treatment } \\
(n=162)^{2}\end{array}$ & $\begin{array}{l}\text { Dutch reference } \\
\text { population (13) } \\
\quad(n=750)\end{array}$ \\
\hline \multicolumn{4}{|l|}{ Mobility (\%) } \\
\hline No problems & 66.2 & 61.4 & 95.1 \\
\hline Some problems & 32.8 & 38.0 & 4.7 \\
\hline Confined to bed & 1.0 & 0.6 & 0.2 \\
\hline \multicolumn{4}{|l|}{ Self-care (\%) } \\
\hline No problems & 93.1 & 93.0 & 96.7 \\
\hline Some problems & 6.9 & 6.4 & 2.9 \\
\hline Unable to & 0.0 & 0.6 & 0.3 \\
\hline \multicolumn{4}{|l|}{ Usual activities (\%) } \\
\hline No problems & 33.7 & 59.4 & 85.8 \\
\hline Some problems & 59.9 & 37.5 & 11.7 \\
\hline Unable to & 6.4 & 3.1 & 2.4 \\
\hline \multicolumn{4}{|l|}{ Pain/discomfort (\%) } \\
\hline No pain/discomfort & 29.7 & 42.0 & 68.0 \\
\hline Moderate pain/discomfort & 62.9 & 49.7 & 30.5 \\
\hline Extreme pain/discomfort & 7.4 & 8.3 & 1.5 \\
\hline \multicolumn{4}{|l|}{ Anxiety/depression (\%) } \\
\hline Not anxious/depressed & 46.0 & 68.2 & 83.6 \\
\hline Moderately anxious/depressed & 47.5 & 28.7 & 15.7 \\
\hline Extremely anxious/depressed & 6.4 & 3.2 & 0.7 \\
\hline EuroQol VAS (mean \pm S.D.) & $66.6 \pm 17.9$ & $71.4 \pm 18.9$ & $85.3 \pm 8.3$ \\
\hline
\end{tabular}

${ }^{1}$ Two missing values; ${ }^{2}$ one missing value.

patients scored below 50 points for visual functioning and appearance respectively.

Several clinical characteristics of the patients were related to the HRQL scores. Non-smokers had higher scores on the GO-QOL visual functioning scale compared with smokers and ex-smokers (83.6 vs 78.0 and 78.4 points respectively). Ex-smokers had higher scores on the GO-QOL appearance scale compared with smokers and non-smokers (83.4 vs 73.7 and 76.5 points). There were no significant differences between the smoking groups on the general HRQL scales.

Patients who were treated with radiotherapy scored on average 3-15 points lower on the HRQL scales compared with patients who were treated with prednisone, except for the scales 'vitality' and 'appearance' on which they scored 1 and 5 points higher. Patients who were treated with both immunosuppression modalities scored mostly in between the other two groups.

The 154 patients who visited the Orbital Centre were classified in responders and non-responders based on their GO severity before the start of the immunosuppressive treatment and after their final treatment. An improvement in visual acuity, in eye muscle motility or diplopia, a decrease of at least $3 \mathrm{~mm}$ in proptosis, and an improvement in soft tissue involvement were considered criteria for response. Eighty-two per cent of the patients were classified as responders. A classification as a non-responder was mostly the result of a decrease in visual acuity. The responders scored on average 19 points higher on visual functioning and 5 points higher on appearance compared with the nonresponders. The responders scored on average 3-13 points higher on the general HRQL scales compared with the non-responders.

Patients with current diplopia (45\%) scored on average 6 and 7 points lower on the GO-QOL visual functioning and appearance respectively compared with patients without diplopia. Patients with current diplopia also scored on average 1-7 points lower on the general HRQL scales compared with patients without diplopia. Patients with a current proptosis of at

Table 3 GO-specific quality of life scores: the GO-QOL (mean \pm S.D.)

\begin{tabular}{lcccc}
\hline & $\begin{array}{c}\text { Go patients before } \\
\text { treatment (3) } \\
(n=70)\end{array}$ & $\begin{array}{c}\text { Go patients during } \\
\text { treatment (11) } \\
(n=206)\end{array}$ & $\begin{array}{c}\text { Go patients mixed } \\
\text { sample (14) } \\
(n=93)\end{array}$ & $\begin{array}{c}\text { Go patients after } \\
\text { treatment } \\
(n=163)\end{array}$ \\
\hline Visual functioning & $54.7 \pm 22.8$ & $58.2 \pm 27.5$ & $65.6 \pm 26.1$ & $78.2 \pm 23.7^{1}$ \\
Appearance & $60.1 \pm 24.8$ & $57.8 \pm 25.4$ & $61.0 \pm 27.4$ & $77.2 \pm 22.3^{1}$ \\
\hline
\end{tabular}

\footnotetext{
${ }^{1}$ Seven and four missing values for visual functioning and appearance respectively.
} 
least $23 \mathrm{~mm}(22 \%)$ scored on average 4 points lower on appearance compared with patients with a current proptosis of less than $23 \mathrm{~mm}$, but the differences on the other HRQL were small and inconsistent.

\section{Discussion}

To our knowledge, we are the first to present data on the long-term effects of GO on HRQL, using validated questionnaires. We showed that GO patients still experience marked limitations in physical and mental functioning, many years after treatment for their GO. The most important problems were related to visual functioning, psychosocial functioning as a consequence of a changed appearance, physical functioning in general, and general health perceptions.

Several issues should be considered when interpreting our results. First, since our study was performed as a satellite project of another study, we included only patients who were treated with radiotherapy and/or prednisone in the AMC between 1982 and 1992. We did not include patients who were referred to our clinic but did not receive radiotherapy or prednisone, nor did we include patients who were treated with radiotherapy or prednisone outside the AMC. This implies that we need to be cautious if we want to generalise our results to all GO patients who were treated with radiotherapy or prednisone or to all GO patients in general in The Netherlands or outside. The HRQL scores of our cohort may be worse than those of these two larger cohorts because we included relatively worse patients in a tertiary referral centre.

Secondly, we did not perform a longitudinal study, but we compared the HRQL scores of our patients with those of other patients who completed the HRQL questionnaire before or during treatment. Unfortunately, HRQL questionnaires were not used or did not exist (GO-QOL) 12 years ago. We do not know if our follow-up cohort was comparable with the newly diagnosed patients when they were diagnosed 12 years ago. And vice versa, we do not know if the patients who are being treated nowadays will be comparable with our follow-up cohort 12 years from now. The HRQL of GO patients who are currently being treated might be better 12 years from now than the HRQL scores of our follow-up cohort because of improved treatment modalities. A proper longitudinal follow-up study should be performed, but this will take many years because validated HRQL questionnaires have only been used in GO patients for a few years $(2,3)$.

Thirdly, the follow-up period was very wide (7-23 years) and one may argue that HRQL was not assessed at comparable times after receiving treatment. Our primary aim was, however, to quantify residual HRQL effects of GO after treatment was considered to be finished, which was the case in all patients.
Another possible bias may have been caused by the relatively old age (mean 58 years vs 48 years for the reference population) and low education (50\% primary education versus $13 \%$ in the reference population) of our group, both factors that are associated with decreased HRQL scores $(10,15)$. It was not possible to adjust the Z-scores simultaneously for gender, age and education because these scores were not available for the reference population. It is conceivable that these differences can explain part of the differences in general HRQL scores between our patients and the reference populations. However, based on the clinical examination, it was clear that our patient group still has a considerable number of symptoms related to GO (Table 1). More than half of the patients had symptoms of diplopia and $28 \%$ had a low visual acuity. Sixty per cent had a proptosis of at least $20 \mathrm{~mm}$. Our previous studies have shown that these symptoms have a profound negative effect on general and disease-specific HRQL $(2,3,11)$. Also in this study, these associations were found (data not shown). The effects of GO symptoms on HRQL might even be larger in older patients, because older patients are less capable of adjusting to their illness, and might thus experience more functional limitations from the same amount of symptoms compared with younger patients.

The clearest indication of a decreased HRQL due to residual GO symptoms is obtained from the results of the GO-QOL questionnaire. Because the GO-QOL specifically measures limitations in functioning as a result of GO, 'healthy' persons should score 100 points (no limitations). Although our patients scored considerably higher (17-23 points) than newly diagnosed patients before treatment, 57 and $61 \%$ of the patients scored below 90 points, which can be considered an important decrease in HRQL (11). Mean scores in this patient group were 77 and 78 points for the two subscales. A score of 75 points is obtained when a patient is a little limited in four of the eight items, or seriously limited in two items (or by other possible combinations). As an example, the 'average patient' in this study is seriously limited in driving and reading because of diplopia and/or decreased visual acuity. In addition, they sometimes feel stared at in the street, sometimes people react unpleasantly, and they still feel very much socially isolated as a result of their changed appearance.

Our results underscore the results of the follow-up study of Bartley et al. (4), who found that $52 \%$ of their respondents felt that their eyes were still abnormal. In addition, Fahrenfort et al. (16) found a number of complaints in Graves' patients who were euthyroid for at least a year, although the validity of their questionnaire should be questioned.

In summary, although our study was cross-sectional in design and can be criticised in several ways, our data are unique and our findings very important. 
We conclude that GO has a marked negative effect on HRQL, even many years after treatment. Symptoms and signs of GO apparently persist and patients keep having trouble with daily visual and general physical functioning. In addition, the negative psychosocial consequences of a changed appearance are still present. These findings suggest that GO should be considered a chronic disease. Aftercare is needed for these patients after their immunosuppressive and surgical treatments.

\section{Acknowledgements}

We would like to thank Mrs W C A M Ompi, Mr S Rijsen, and Mrs J A M Spittelen for their technical assistance in the outpatient clinic and secretarial work for this study.

\section{References}

1 Burch HB \& Wartofsky L. Graves' ophthalmopathy: current concepts regarding the pathogenesis and management. Endocrine Reviews 199314 747-793.

2 Gerding MN, Terwee CB, Dekker FW, Prummel MF \& Wiersinga WM. Quality of life in patients with Graves' ophthalmopathy is markedly decreased: measurement by the Medical Outcome Study instrument. Thyroid 19977 885-889.

3 Terwee CB, Gerding MN, Dekker FW, Prummel MF \& Wiersinga WM. Development of a disease-specific quality of life questionnaire for patients with Graves' ophthalmopathy: the GO-QOL. British Journal of Ophthalmology $1998 \mathbf{8 2} 773-779$.

4 Bartley GB, Fatourechi V, Kadrmas EF, Jacobsen SJ, Ilstrup DM, Garrity JA et al. Long-term follow-up of Graves' ophthalmopathy in an incidence cohort. Ophthalmology 1996103 958-962.

5 Ware JE Jr \& Sherbourne CD. The MOS3.6-item Short-Form Health Survey (SF-36). I. Conceptual framework and item selection. Medical Care $199230473-483$.

6 The EuroQol Group. EuroQol - a new facility for the measurement of health-related quality of life. Health Policy 199016 $199-208$.
7 Greenfield S, Aronow HU, Elashoff RM \& Watanabe D. Flaws in mortality data. The hazard of ignoring comorbid disease. Journal of the American Medical Association $19882602253-2255$.

8 Mourits MP, Prummel MF, Wiersinga WM \& Koornneef L. Clinical Activity Score as a guide in the management of patients with Graves' ophthalmopathy. Clinical Endocrinology 1997 47 9-14.

9 Bergner M, Bobbitt RA, Kressel S, Pollard WE, Gilson BS \& Morris JR. The Sickness Impact Profile: conceptual formulation and methodology for the development of a health status measure. International Journal of Health Services 19766 393-415.

10 Aaronson NK, Muller M, Cohen PDA, Essink-Bot ML, Fekkes M, Sanderman R et al. Translation, validation, and norming of the Dutch language version of the SF-36 health survey in community and chronic disease populations. Journal of Clinical Epidemiology 199851 1055-1068.

11 Terwee CB, Dekker FW, Mourits MP, Gerding MN, Baldeschi L, Kalmann $\mathrm{R}$ et al. Interpretation and validity of changes in scores on the Graves' Ophthalmopathy Quality of Life questionnaire (GO-QOL) after different treatments. Clinical Endocrinology 200154 391-398.

12 Ware JE, Kosinski M \& Keller SD. SF-36 Physical and Mental Health Summary Scales: a User's Manual. Boston, MA: The Health Institute, 1994.

13 Essink-Bot ML, Stouthard M \& Bonsel GJ. Generalizability of valuations on health states collected with the EuroQol questionnaire. Health Economics 19932 237-246.

14 Terwee CB, Gerding MN, Dekker FW, Prummel MF, van der Pol JP \& Wiersinga WM. Test-retest reliability of the GO-QOL: a diseasespecific quality of life questionnaire for patients with Graves' ophthalmopathy. Journal of Clinical Epidemiology $1999 \mathbf{5 2}$ $875-884$.

15 Glasgow RE, Ruggiero L, Eakin EG, Dryfoos J \& Chobanian L. Quality of life and associated characteristics in a large national sample of adults with diabetes. Diabetes Care 199720 562-567.

16 Fahrenfort JJ, Wilterdink AML \& van der Veen EA. Long-term residual complaints and psychosocial sequelae after remission of hyperthyroidism. Psychoneuroendocrinology 200025 201-211.

Received 18 December 2001

Accepted 11 March 2002 